\title{
O ENSINO DA TÉCNICA DE CURATIVO E RETIRADA DE PONTOS EM INCISÃO CIRÚRGICA ATRAVÉS DA ESTRATÉGIA DE SIMULAÇÃO*
}

\author{
Edna Mara Ferreira do Nascimento** \\ Maria Lúcia Brandão Cançado**
}

\begin{abstract}
RESUMO: As autoras apresentam um método de ensino das técnicas de curativo e retirada de pontos, através da estratégia de simulação. Descrevem a montagem do dispositivo utilizado, o qual possibilita o desenvolvimento das habilidades psicomotoras necessárias para a realização desses procedimentos. Tecem considerações sobre a importância e necessidade de intermediar, em ambiente laboratorial, o ensino teórico-prático das técnicas de enfermagem.
\end{abstract}

ABSTRACT: The authors present a teaching method of dressing change and suture removol using simulation strategies. They describe how to assemble the employed device which simulates a surgical wound and allows the development of psycomotor skills required to execute these procedures in the clinical situation. They are also concerned about the necessity of intermediate theoretical and practical teaching of nursing techniques in laboratory settings.

\section{INTRODUÇÃO}

A realização do curativo*** é, com frequência, momento de grande expectativa para o paciente que apresenta uma ferida cirúrgica. ${ }^{(1)}$ Ele deseja tomar conhecimento do aspecto da incisão, preocupa-se com a evolução da cicatrização e teme sentir dor durante o procedimento do curativo e retirada de pontos. Quando se trata do primeiro curativo após a cirurgia, tal expectativa é ainda maior, uma vez que o paciente ainda desconhece o tamanho da incisão cirúrgica.

Para os integrantes da equipe de saúde, habituados com a rotina do pós-operatório, podem parecer exageradas as reações de medo e as queixas de dor por parte do paciente, mas é necessário entender esta situação, considerando que a hospitalização, por si só, representa um período de crise para o homem. Quando hospitalizado, o homem tem sua capacidade de auto-determinação afetada, porque torna-se dependente da equipe assistencial, principalmente quando a sua doença requer tratamento cirúrgico, pois a insegurança e o temor assumem maiores dimensões. ${ }^{(2)}$

Manter essa situação sob controle é, em grande parte, papel da enfermagem, através da interação com o paciente, iniciada no momento da internação e fortalecida susequentemente, esclarecendo seus problemas e aliviando a ansiedade natural.

O papel da enfermagem assume grande importância no momento da realização do curativo. Faz parte desse procedimento, considerar as impressões manifestadas pelo paciente em relação à incisão cirúrgica, o que significa interpretar, com segurança, atitudes do paciente que supervalorizem ou substimem a situação.

Demonstrar conhecimento e habilidade técnica específica na realização do curativo, também contribuem para diminuir o nível de ansiedade do paciente.

FRIEDLANDER $^{(5)}$ destaca a crescente importância dos direitos do paciente em receber uma assistência segura e eficiente e que, nesse contexto, a

* Prêmio Zaira Cintra Vidal - $2^{\circ}$ lugar - 44 Congresso Brasileiro de Enfermagem - Brasília - D.F. 4 a 9 de outubro de 1992.

** $\quad$ Professoras Assistentes do Departamento de Enfermagem da Universidade Federal de Mato Grosso.

*** Curativo é o tratamento de uma ferida, com a finalidade de protegê-la, mantendo-a seca e limpa; comprimi-la se necessário; proporcionar conforto psicológico e físico para o paciente; imobilizar a área afetada; e favorecer a aplicação de medicamentos.(2) 
enfermagem deve discutir e preocupar-se cada vez mais com a segurança dos serviços que presta.

Buscar formas de maximizar a eficiência do processo de ensino/aprendizagem nas dimensões que constituem a formação do profissional adequado às necessidades da sociedade, deve ser preocupação constante do professor.

Considerando que o paciente: necessita sentir-se seguro em relação aos cuidados com a ferida operatória; que atribui à habilidade técnica do executante do curativo importante fator de segurança; e ainda, que os procedimentos envolvidos na assepsia cirúrgica requerem acentuado detalhadamento dos passos do mesmo em função das graves consequências que podem advir da contaminação, julgamos ser ideal o ensino através da simulação.

O método de ensino apresentado neste trabalho é de fácil operacionalização, simula a realização de curativos e retirada de pontos de uma forma bastante semelhante à realidade, não implicando necessariamente, na existência de laboratório de enfermagem propriamente dito, para sua utilização.

\section{OBJETIVOS DO MÉTODO}

Objetivo Geral - Oportunizar ao aluno, o desenvolvimento de habilidades psicomotoras para a realização de curativos e retirada de pontos de incisões cirúrgicas em laboratório.

\section{Objetivos Específicos:}

1. Conhecer materiais e soluções utilizadas no procedimento.

2. Preparar o material necessário para o procedimento.

3. Utilizar o material considerando as técnicas de manuseio de material esterilizado.

4. Realizar curativos em incisões cirúrgicas localizadas em diferentes regiões da superfície corporal.

5. Retirar pontos da pele nas diferentes formas possíveis.

6. Fazer anotação de enfermagem referente ao procedimento.

\section{REVISÃO DA LITERATURA}

Para FEIX ${ }^{(3),}$ hospitalização e cirurgia são condições que influem no comportamento do indivíduo., pois afetam o sistema de vida.

A problemática sentida pelo paciente com pers- pectiva de cirurgia, consiste de questões relacionadas à sobrevivência, à dor, ao medo da anestesia, ao desconhecido, à possibilidade de morte durante ou após a cirurgia, de não ser operado pelo "seu" médico, a problemas econômicos e familiares e à recuperação e convalescença, incluindo os cuidados requeridos pela mesma. ${ }^{(2)}$

Sinais de recuperação e preocupação com a integridade física são evidenciados, em intensidades variadas, em todos os pacientes, mesmo naqueles com experiências anteriores ou adequadamente preparados para a cirurgia.

Sinais de recuperação e preocupação com a integridade física são evidenciados, em intensidades variadas, em todos os pacientes, mesmo naqueles com experiências anteriores ou adequadamente preparados para a cirurgia.

Passado o período pré-operatório, desaparecem parte das preocupações e continuam ou se intensificam aquelas relacionadas ás possíveis complicações pósoperatórias e dentre elas, salientamos ás relacionadas á incisão cirúrgica, que são: a insegurança com relação á eficácia da sutura, principalmente na movimentação e deambulação, receio de que venha ocorrer infecção pós-cirúrgica e o medo da dor durante o curativo.

Segundo FEIX $^{(3)}$, a expectativa em relação ao primeiro curativo foi referida por $61 \%$ dos pacientes de sua pesquisa, como sendo a "habilidade técnica específica do profissional".

Para Magill apud FRIEDLANDER ${ }^{(6)}$, a habilidade motora precisa ser aprendida para ser executada correta e eficientemente.

Para a dimensão do ensino da enfermagem que envolve a abordagem mais restrita da profissão, e, nesse contexto estão incluídos os procedimentos de enfermagem amplamente realizados por todos os elementos de sua equipe, consideramos ser a simulação dos procedimentos em ambiente laboratorial ou mesmo improvisado, um dos recursos que favorecem o êxito na aprendizagem sem estresse e riscos, tanto para o paciente como para o aluno.

FRIENDLANDER $^{(6)}$, revendo a literatura do último ano, principalmente americana, verificou que o uso do laboratório de enfermagem para a aprendizagem e desenvolvimento de habilidades para executar procedimentos de enfermagem é defendido por argumentos de natureza ética, psicológica, econômica e pedagógica.

\section{APRESENTAÇÃO DO MÉTODO}

O método utiliza um dispositivo que simula a pele 
suturada, confeccionado com um pedaço de faixa de Smarch de aproximadamente $20 \mathrm{~cm} \times 6 \mathrm{~cm}$ sobre o qual é feito um traço com caneta (simulando a incisão). Ao longo da "incisão", são passados pontos de algodão 00 ou 000 , simulando uma sutura (anexo: figuras 1 e 2).

Este dispositivo é fixado com fita adesiva cirúrgica cor da pele (tipo Micropore ${ }^{\circledR}$ ) sobre a pele (anexo figura 3), em diferentes regiões do corpo (abdome, membros superiores ou inferiores) de um aluno ou manequim de técnicas.

Nesse dispositivo fixado na pele, é realizado o procedimento de curativo conforme se faz na realidade (anexo: figuras 4 e 5).

Quando o dispositivo é colocado em membros inferiores ou superiores, a fixação do curativo pode ser feita através de enfaixamento com atadura de crepe, oportunizando, dessa forma, o desenvolvimento da técnica de bandagem.

A retirada de pontos, alternados ou totais, é feita como na realidade, inclusive utilizando as duas formas mais comuns de se cortar o ponto, ou seja, com lâmina de bisturi, ou com tesoura esterilizada (figuras 6 e 8). Após a finalização da técnica (figura 8), segue-se à anotação de enfermagem.

A demonstração da técnica é primeiramente realizada pelo professor, e a seguir, cada aluno tem a oportunidade de repetir o procedimento sob a orientação do docente (e eventualmente, do monitor da disciplina) e sob a observação dos colegas. Vários dispositivos podem ser montados para possibilitar a aprendizagem de vários alunos simultaneamente. Um roteiro auto-instrucional, pode ser utilizado pelo aluno, permitindo repetições do procedimento e desa forma, auxiliando a memorização dos passos.

O método descrito vem sendo utilizado nos últimos semestres nas disciplinas de Introdução à Enfermagem e Introdução à Prática Hospitalar pertencen- tes, respectivamente, aos cursos de graduação em Enfermagem e Medicina da Universidade Federal de Mato Grosso.

Como estratégia de ensino tem atingido o objetivo de oportunizar o desenvolvimento de habilidades psicomotoras para realização desse procedimento, conforme avaliações de professores e de alunos. A grande vantagem da mesma é a possibilidade de intermediar teoria e prática, auxiliando portanto, a transposição da barreira existente entre essas duas dimensões do ensino em enfermagem. ${ }^{(7)}$

\section{CONSIDERAÇÕES FINAIS}

Oportunizar ao aluno o desenvolvimento de habilidades psicomotoras imprescindíveis para o desempenho profissional sem reduzir o cuidado da ferida incisional à uma mera sucessão de passos ou à sua automatização. Esta é a forma pela qual os docentes da disciplina de Introdução à Enfermagem da Universidade Federal de Mato Grosso têm utilizado e chegado a bons resultados no ensino/aprendizagem desse procedimento.

A ênfase do conhecimento científico, que norteia o cuidado qualificado, e a pesquisa, que busca novas tecnologias, através das quais o processo de reparação de tecidos possa ser facilitado e os que a prejudicam possam ser evitados, devem constituir o objetivo maior do plano de ensino.

$\mathrm{O}$ argumento da falta de condições ideais de ensino e campos de prática não pode justificar um ensino de enfermagem deficiente. Segundo FRIEDLANDER. ${ }^{(4)}$ "uma estratégia instrucional de alta qualidade não é sinônimo de sofisticação, mas o resultado de um planejamento cuidadoso, adequado e cientificamente implementado, e, ainda, que em um país com carência de recursos como o Brasil, deve-se tentar substituir a sofisticação pela criatividade".

\section{REFERÊNCIAS BIBLIOGRÁFICAS}

1. ATKINSON, L.D. \& MURRAY. ME. Fundamentos de Enfermagem. $1^{\text {a }}$ Edição. Rio de Janeiro: Guanabara koogan, 1989

2. FARO, A.C.M.E. Plantas medicinais: um auxílio para a cicatrização. Acta Paul. Enf, 1 (2): 73-9. 1988.

3. FEIX, M.A.F. O paciente hospitalizado e a incisão cirúrgica abdominal - problemas, percepção e expectativas. Dissertação de Mestrado - Escola de Enfermagem da Universidade de São Paulo, 1984.
4. FRIEDLANDER, M.R. O ensino dos procedimentos básicos no Laboratório de Enfermagem: comparação entre dois métodos de instrução. Tese de Doutorado - Escola de Enfermagem da Universidade de São Paulo, 1984.

5. FRIEDLANDER, M.R. Vantagens do ensino em laboratório. São Paulo, 1991 (mimeo).

6. Habilidades psicomotoras de enfermagem: defỉnição de termos. Rev. Esc. Enf. USP (in press).

7. NASCIMENTO, E.M.E. \& CANÇADO, M.L.B. O uso de um sistema artesanalmente construído para o ensino da punção e infusão venenosa. Rev. Bras. Enf. 1991; 144(4): 18-21. 


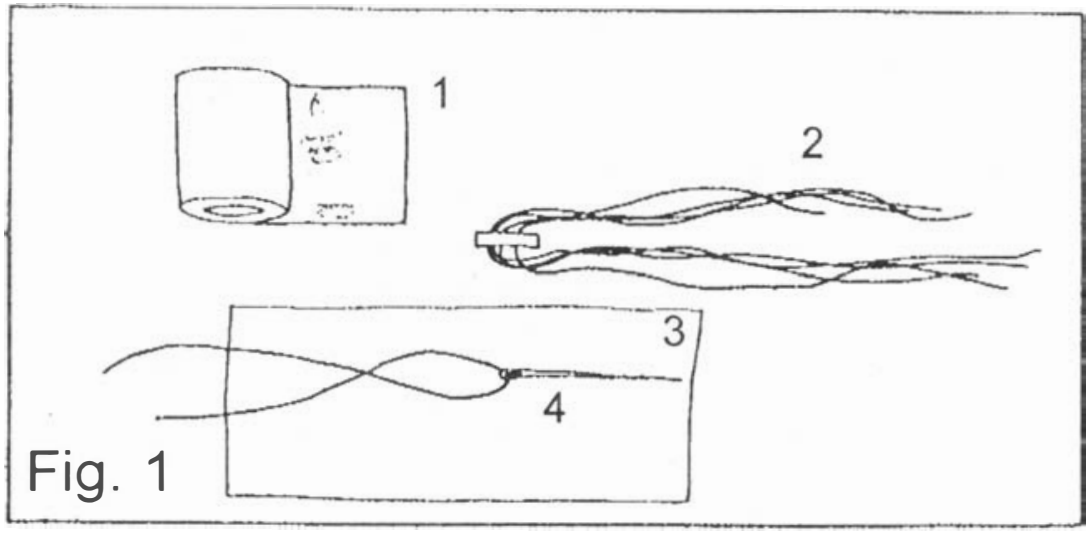

1- Fita adesiva cirúrgica

3- Faixa de Smarch

2- Fio de algodão $\mathrm{p} /$ sutura

4- Agulha

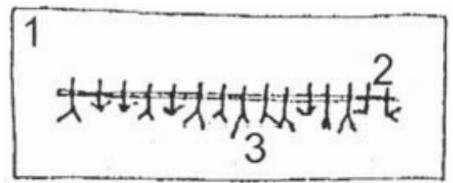

Fig. 2

1- Fita de Smarch

2- Traço ("incisão cirúrgica")

3- Pontos de algodão

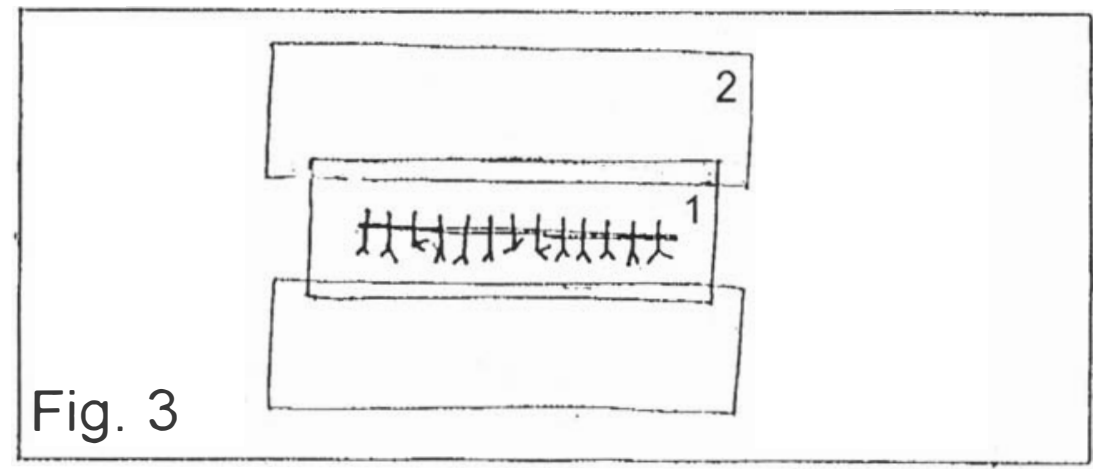

1- Dispositivo Instalado

2- Fita adesiva cirúrgica 


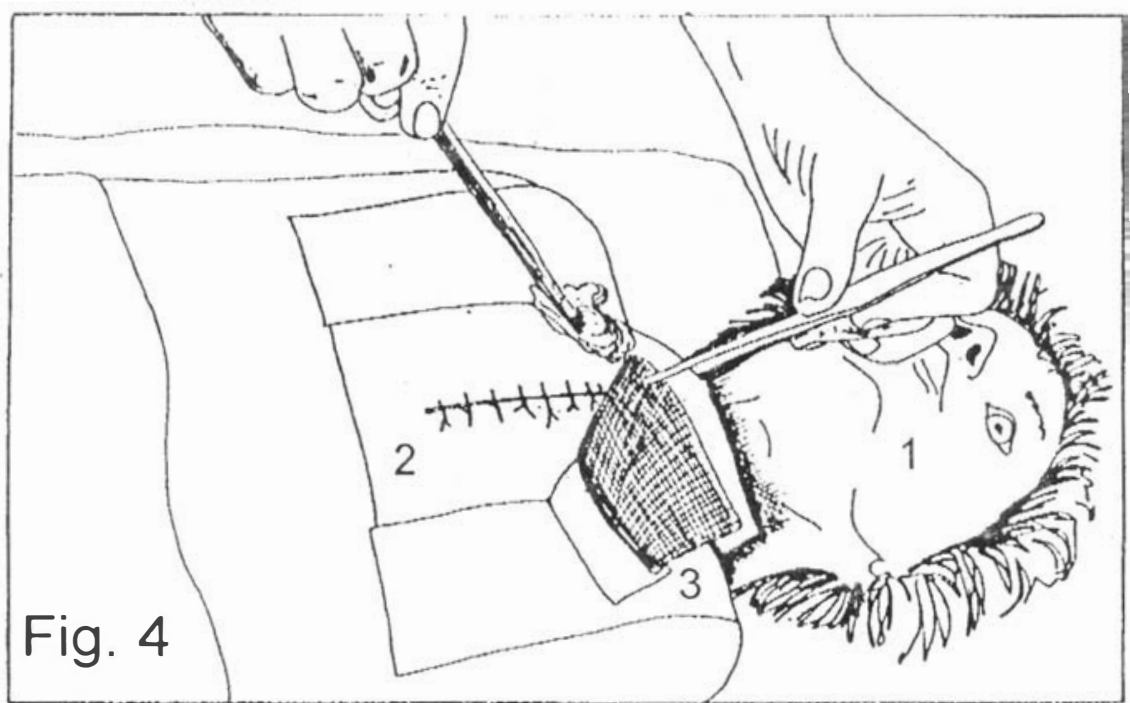

1- Manequim de técnicas

2- Dispositivo

3- Esparadrapo e gazes sendo retirados

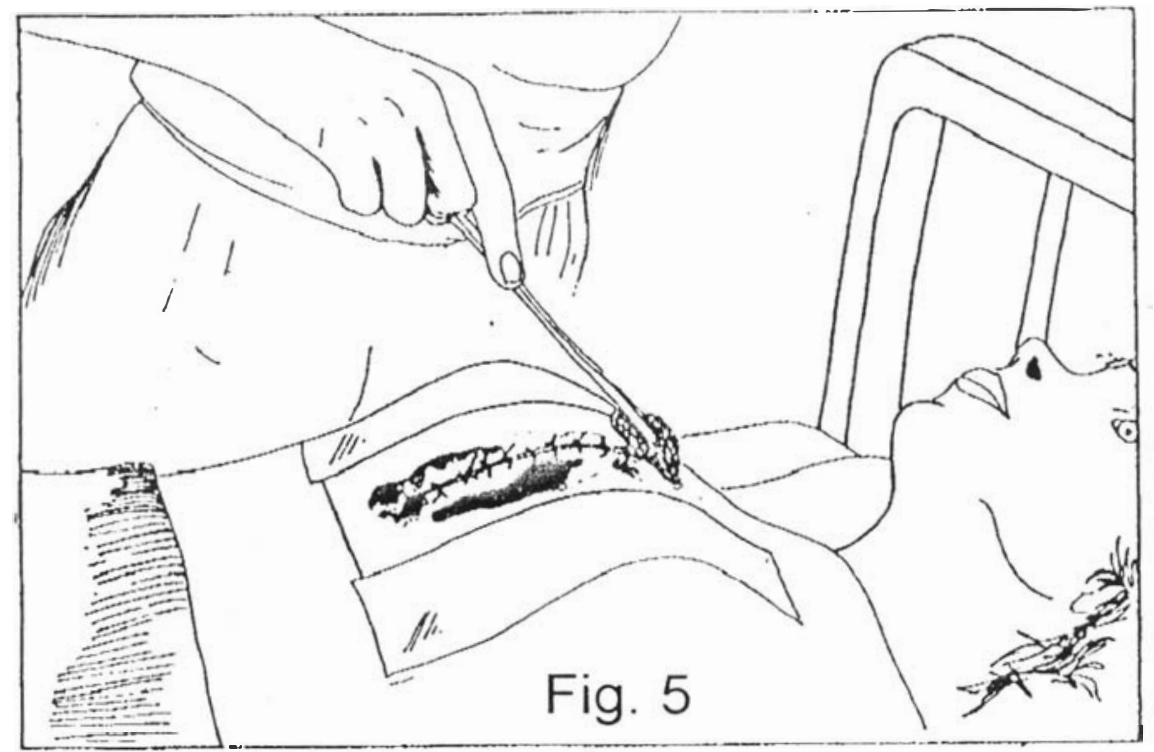

Limpeza e Antissepsia da "incisão cirúrgica" 


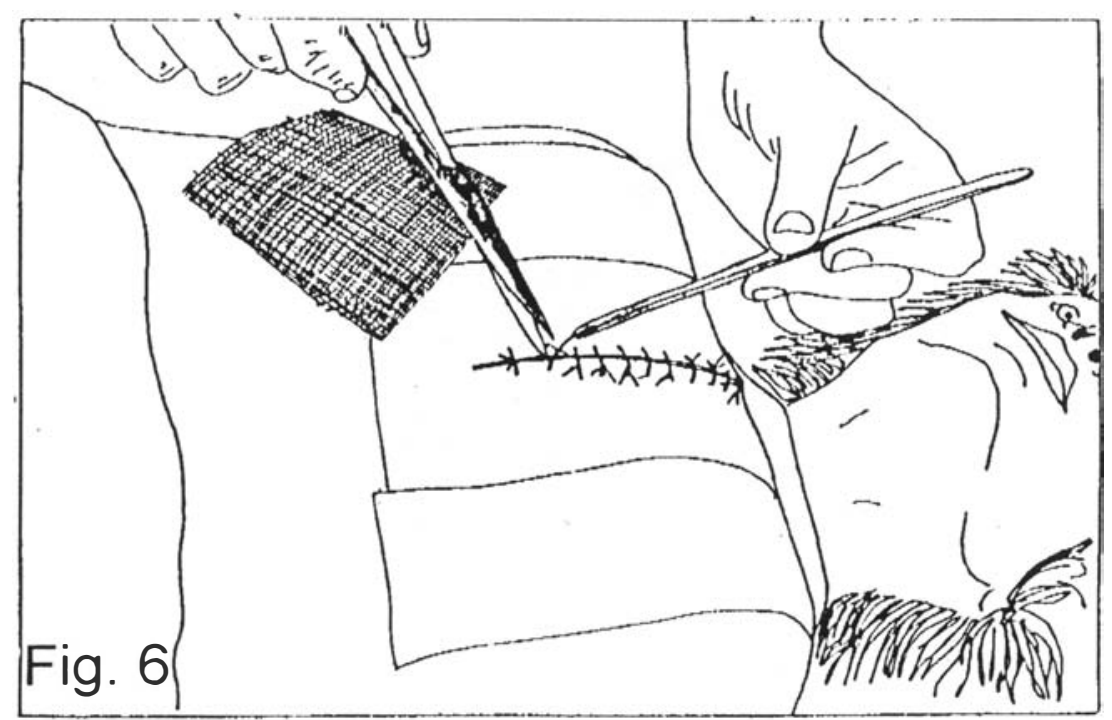

Retirada de pontos com tesoura

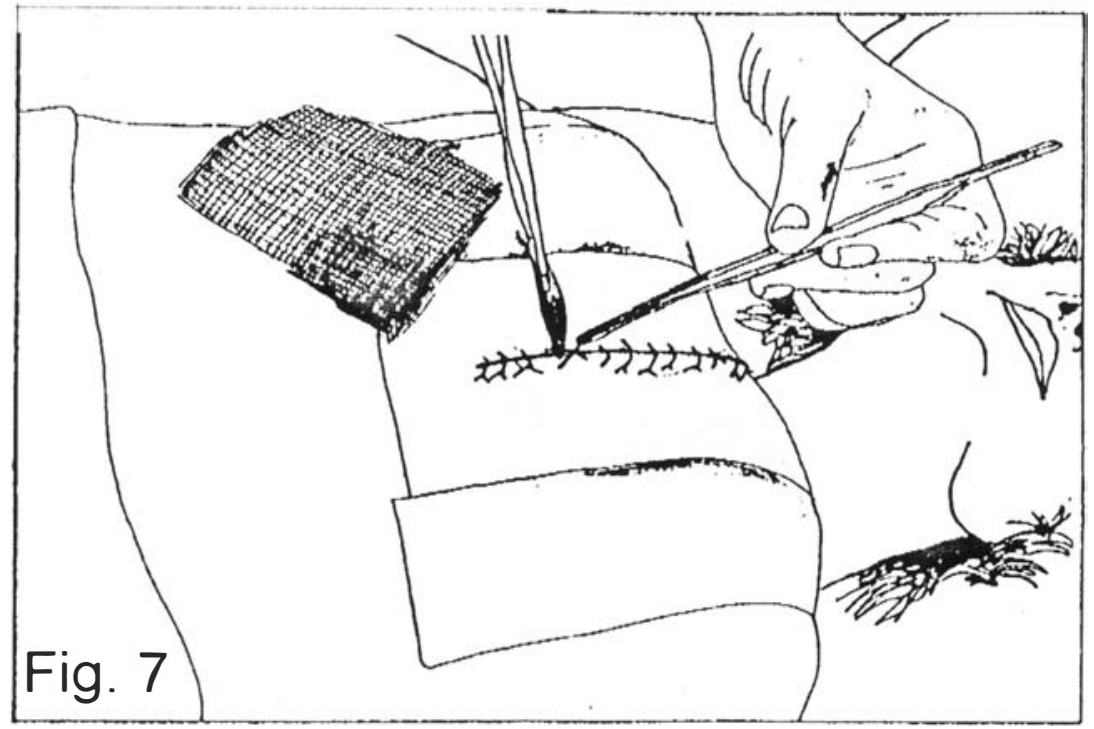

Retirada de pontos com bisturi 


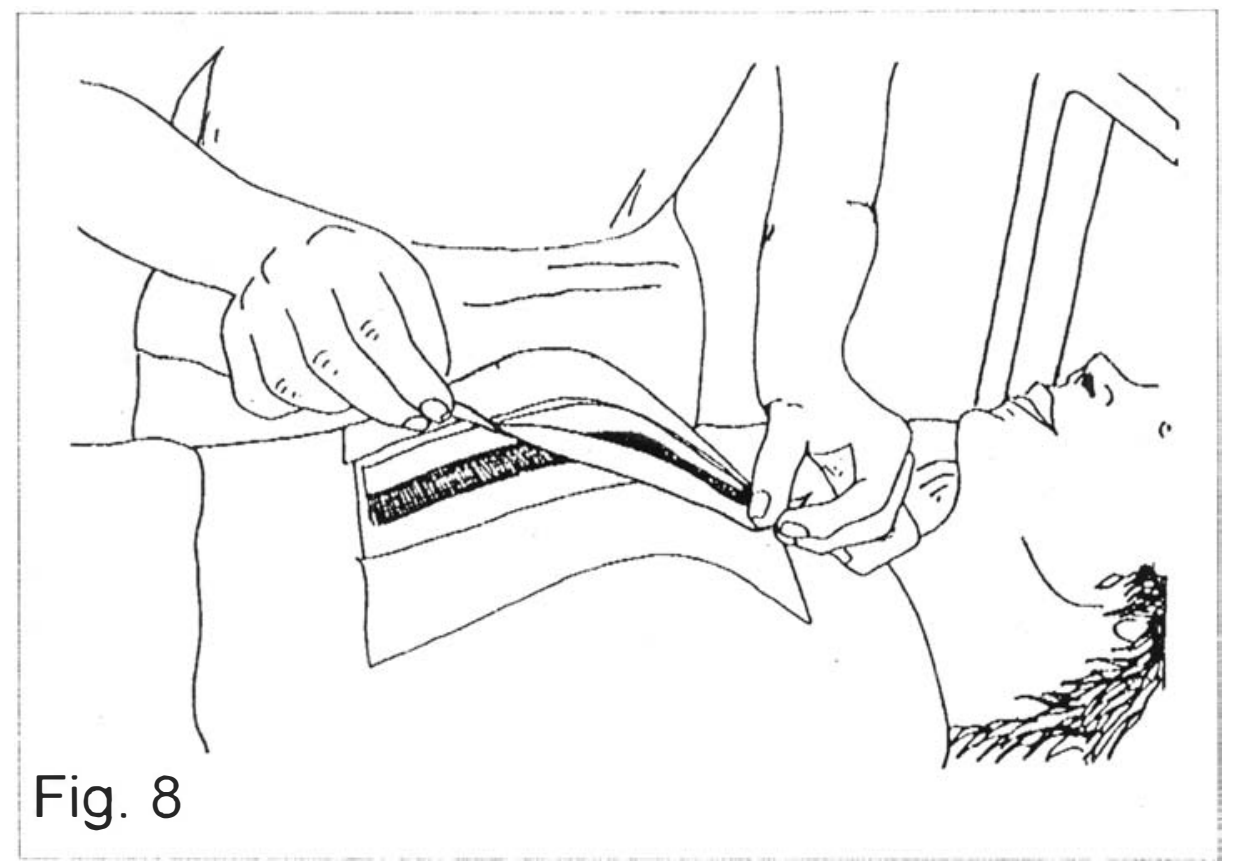

Finalização do curativo 